\title{
Zero-Mass Limit in Perturbative Quantum Field Theory
}

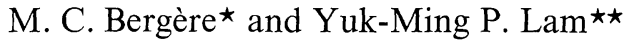 \\ Service de Physique Théorique, Centre d'Etudes Nucléaires de Saclay, F-91190 Gif-sur-Yvette, France
}

\begin{abstract}
A new R-operation which satisfies Bogolubov-Parasiuk and Hepp recurrence and which is infrared and ultra violet convergent graph by graph, is defined in perturbative quantum field theory. This new subtraction scheme is used to achieve the zero-mass limit of a massive field theory.
\end{abstract}

\section{Introduction}

In 1970 Callan [1] and Symanzik [2] introduced a differential equation to study the high energy behaviour of renormalized vertex functions in Lagrangian field theory. By homogeneity arguments, the asymptotic high energy behaviour of these functions also describes their infrared limits when all masses $m_{i}$ in the theory tend to zero. For strictly renormalized field theory, it is found that such infrared limits exist at nonexceptional momenta provided that the divergent vertex functions are subtracted in a convenient fashion [3]. This result was also proved by Gell-Mann and Low using the technique of the renormalization group [4].

This paper intend to show the detailed mechanism of the infrared limit. In a recent publication [5] we have obtained explicitely the asymptotic high energy behaviour of a renormalized Feynman amplitude subtracted at zero momentum; we have especially given specific "geometric" rules to construct the coefficients of all powers of logarithm for the leading power behavior. These rules are given at the end of this introduction for the case of graphs generated by a strictly or non renormalizable field theory at non-exceptional momenta. In that case, the structure of these coefficients is such, that there exists for any graph a linear combination of Feynman amplitudes which has a zero-mass limit. Namely, the powers of logarithm which arise in the zero-mass limit of each Feynman amplitude when subtracted at zero momentum, are cancelled by the logarithms which enter the coefficients of the linear combination. It is the purpose of Section II to show this

* Chargé de recherche CNRS. - Work supported in part by Alexander Von Humboldt fellowship. $\star \star$ Work supported in part by Deutsche Forschungsgemeinschaft. - Institut für theoretische Physik, Freie Universität Berlin, Berlin, Federal Republic of Germany, and present address: Radiological Research Laboratory, Columbia University, 630 West 168 St. New York, New York 10032, USA. 
result. The next question which has to be solved is the following one: how is, this finite combination of Feynman amplitudes attached to each graph, going to break unitarity, when we sum over all graphs in a vertex function? In fact, it does not break unitarity because it exists a Lagrangian with real, infrared divergent counterterms, which generates this combination of amplitudes. In Section III, we illustrate this property in the simple case of a $\phi^{4}$ type theory. Then, it is shown in Section IV that the infrared divergent counterterms can be avoided by introducing a new $R$-operation. This $R$-operation, which is defined here in the $\alpha$-parameter space and which seems similar to Lowenstein and Zimmermann [6] $R$-operation defined in momentum space, satisfies Bogolubov and Parasiuk [7] and Hepp [8] (BPH) recurrence over generalized vertices. This subtraction procedure has the property to give an infrared and ultraviolet finite amplitude for all graphs generated in a strictly or non renormalizable field theory at non exceptional momenta. Finally, an Appendix is devoted to the structure of the vertex and other useful functions expressed in terms of the counterterms chosen in the Lagrangian. The entire work is performed in Euclidian space; the results can be applied in Minkowski space in the sense of distributions (we insist upon the fact that all masses tend to zero to avoid certain types of exceptional momenta due to Minkowski space).

Let us now remind to the reader, the rules obtained in Ref. [5] for the asymptotic high energy behaviour of a single Feynman amplitude.

Definition 1. Given a graph $G$ with non zero external momentum $\left(p_{1}, \ldots, p_{n}\right)$ $n \geqq 2$, and satisfying the overall momentum conservation law $\sum_{i=1}^{n} p_{i}=0$, a subgraph $\mathscr{S} \subseteq G$ is said to be essential if its reduced subgraph $[G / \mathscr{S}]$ has zero external momenta at all its vertices.

We must note that the essentiality of a subgraph depends upon the fact that the external momenta are exceptional (partial sum equal to zero) or not. In Ref. [5], it is shown that the asymptotic high energy behaviour of a Feynman amplitude at non exceptional momenta has a different structure, whether the superficial degree of divergence $\omega(\mathscr{S})$ of any essential connected subgraph $\mathscr{S} \neq G$ is less than the superficial degree of divergence of the graph $G$, or not. In the present work, we consider only the case

$$
\omega(\mathscr{S})<\omega(G)
$$

for all essential connected subgraphs $\mathscr{S} \neq G$. It is easy to see [5] that if, at every vertex, we have

$$
B+(3 / 2) F+d \geqq 4
$$

where $B, F$, and $d$ are respectively the number of boson lines, fermion lines and derivative couplings attached to the vertex, then (I.1) is satisfied. Condition (I.2) implies that we restrict ourselves to strictly $(=)$ or non $(>)$ renormalizable field theory, and that we exclude superrenormalizable vertices and two lines vertices. 
A renormalized, Feynman amplitude expressed in the $\alpha$-parametric form is defined by the integrals [10]

$$
I_{G}(p, m)=\int_{0}^{\infty} \prod_{a=1}^{l} d \alpha_{a} e^{-\sum_{a=1}^{l} \alpha_{a} m_{a}^{2}} R\left\{\pi\left(\frac{-1}{\sqrt{\alpha_{a}}} \frac{\partial}{\partial z_{a}}\right) Z_{G}(\alpha, p, z)\right\}_{z_{a}=0} .
$$

In (I.3), $l$ is the number of lines of the graph $G$, the derivatives $\frac{\partial}{\partial z}$ take cares of derivative couplings and spinor propagators, the function $Z_{G}(\alpha, p, z)$ is given by

$$
Z_{G}(\alpha, p, z)=\left[P_{G}(\alpha)\right]^{-2} e^{a^{\sum_{=1}^{l} z_{a}^{2} / 4}} e^{-\sum_{i, j=1}^{n-1}\left(p_{i}+\sum_{a=1}^{l} \varepsilon_{i a} z_{a} / 2 \sqrt{\alpha_{a}}\right)\left[d G_{G}^{-1}(\alpha)\right]_{t_{j}}\left(p_{j}+\sum_{b=1}^{l} \varepsilon_{j} z_{b} / 2 \sqrt{\alpha_{b}}\right)}
$$

where $P_{G}(\alpha)$ and $\left[d_{G}^{-1}(\alpha)\right]_{i j}$ are the Symanzik functions [9] of the graph $G, n$ is the number of vertices of $G$, and $\varepsilon_{i a}$ is the incidence matrix of $G$. The operator $R$ in (I.3) is a subtraction operator expressed in terms of generalized Taylor operators $[10] \tau$, and which acts upon the $\alpha$-parameters of the subgraphs. This operator ensure the ultraviolet convergence $\left(\alpha_{s}^{\prime} \sim 0\right)$ by subtracting the subgraphs at zero external momenta.

If we consider a graph $G$, its Feynman amplitude can be splitted into two parts

$$
I_{G}(p, m)=I_{G}^{a s}(p, m)+\Delta I_{G}(p, m),
$$

according to the expansion of $I_{G}(p, m)$ around $m=0$, such that

$$
\Delta I_{G}(p, m) \sim m \log ^{x} m,
$$

while $I_{G}^{a s}(p, m)$ diverges when $m \rightarrow 0$.

In Ref. [5], the following rules are given for graphs $G$ satisfying the inequality (I.1), at non exceptional momentum:

Definition 2. A forest $\mathscr{F}$ is a set of subdiagrams such that any subset of mutually noninclusive elements is disjoint.

Definition 3. A generalized vertex is a one-line irreducible, connected, subgraph $\mathscr{S}$, such that any other subgraph with the same vertices as those of $\mathscr{S}$, is contained in $\mathscr{S}$.

Then, $I_{G}^{a s}(p, m)$ diverges logarithmically only and takes the form

$$
I_{G}^{a s}(p, m)=\sum_{\mathscr{F}^{\prime}} \chi_{\mathscr{F}^{\prime}} \prod_{\mathscr{S} \in \mathscr{F}^{\prime}}\left\{2 \tilde{\beta}_{[\mathscr{S} / \operatorname{Max} \mathscr{S}]_{\Lambda(\mathscr{C})}}^{\chi_{\omega(\mathscr{C})}}\right\} \phi_{[G / \operatorname{Max} G]_{\Lambda(G)}}(p, m) .
$$

In (I.7), $\mathscr{F}^{\prime}$ is a forest of $r\left(\mathscr{F}^{\prime}\right)$ divergent generalized vertices different from the graph $G$ itself; the sum runs over all such forests including the empty one. The numerical factor $\chi_{\mathscr{F}}$, is

$$
\chi_{\mathscr{F}^{\prime}}=r\left(\mathscr{F}^{\prime}\right) ! / \prod_{\mathscr{S} \in \mathscr{F}^{\prime}}[v(\mathscr{S})+1]
$$

where $v(\mathscr{S})$ is the number of elements of $\mathscr{F}^{\prime}$ inside $\mathscr{S}$, The subgraph $\left[\frac{\mathscr{S}}{\operatorname{Max} \mathscr{S}}\right]$ is the reduced subgraph obtained from $\mathscr{S}$ when all the subgraphs $\mathscr{S}^{\prime}:\left\{\mathscr{S}^{\prime} \in \mathscr{F}^{\prime}\right.$, $\left.\mathscr{S}^{\prime} \subset \mathscr{S}\right\}$ are contracted into points. The functions $\phi$ are of no use to the present 
work and we refer the reader to Ref. [5] for their definition; let us simply mentionned that $\phi$ vanishes whenever $\mathscr{F}^{\prime}$ contains an essential subgraph. It is the structure of $I_{G}^{a s}(p, m)$ in the coefficients $\tilde{\beta}$ which is most important to understand. These coefficients arises originally in "Zimmermann's identity" which relates oversubtracted mass inserted Feynman amplitudes to minimally subtracted ones.

$$
\sum_{a=1}^{l} m_{a}\left(\partial / \partial m_{a}\right) I_{G}(p, m)+2 \sum_{T} \tilde{\beta}_{T}^{\chi_{\omega(T)}} I_{[G / T] \chi_{\omega(T)}}(p, m)=-2 I_{G}(p, m) .
$$

In (I.9), $m \frac{\partial}{\partial m}$ generates a sum of oversubtracted mass insertions in the amplitude for the graph $G$, while $\tilde{I}_{G}(p, m)$ corresponds to the same sum of amplitudes with minimally subtracted mass insertions; the sum, in the left hand side runs over all divergent generalized vertices $T$ in $G$. Given the minimally subtracted mass inserted amplitude $\tilde{I}_{T}(k, m)$ for the subgraph $T$ with external momenta $k_{i}$, and given a sequence of $\omega(T)$ external momentum $k_{i_{1}}, \ldots k_{i_{\omega(T)}}$ where $\omega(T)$ is the superficial degree of divergence of $T$ and where each momentum $k_{i}$ can be present several times in the sequence, we define

$$
\tilde{\beta}_{T}^{\chi \omega(T)}=\left.\frac{-1}{\omega(T) !} \frac{\partial^{\omega(T)}}{\partial k_{i_{1}} \ldots \partial k_{i_{\omega(T)}}} \tilde{I}_{T}(k, m)\right|_{k_{\imath}=0}
$$

The subgraph $\left[\frac{G}{T}\right]_{\chi \omega(T)}$ is the reduced subgraph obtained from $G$ by contracting $T$ into a point and by attaching to that point a set of derivative couplings defined by the sequence $\chi$. In (I.9), summation over all sequences $\chi$ of $\omega(T)$ external momentum is understood. The coefficients $\tilde{\beta}_{[\mathscr{S} / \operatorname{Max} \mathscr{S}]_{\Lambda(\mathscr{S})}}^{\chi_{\omega} \mathscr{\varphi}_{1}}$ in (I.7) are defined as those in (I.10) except that $\mathscr{S}$ is itself reduced by the contraction into points of several disjoint, maximal subgraphs $\mathscr{S}_{i}^{\prime}:\left\{\mathscr{S}_{i}^{\prime} \in \mathscr{F}^{\prime}, \mathscr{S}_{i}^{\prime} \subset \mathscr{S}\right\}$, and $\Lambda(\mathscr{S})=\bigcup_{i} \chi_{\omega\left(\mathscr{S}_{i}^{\prime}\right)}$. In (I.7) summation between $\Lambda_{s}^{\prime}$ and $\chi_{s}^{\prime}$ is understood.

\section{Infrared Convergent Combination of Feynman Amplitudes}

This combination of Feynman amplitudes is obtained by applying Zimmermann's identity (I.9) recurrently. From (I.5), (I.9), and the power counting theorem for renormalized amplitude $[5,12]$, we have

$$
\sum_{a=1}^{\prime} m_{a}\left(\partial / \partial m_{a}\right) I_{G}^{a s}(p, m)+2 \sum_{T} \tilde{\beta}_{T}^{\chi \omega(T)} I_{[G / T] \chi_{\omega(T)}}^{a s}(p, m)=0
$$

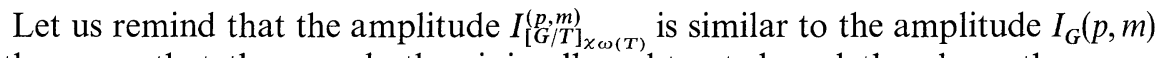
in the sense that they are both minimally subtracted, and they have the same number of external legs and the same superficial degree of divergence. Also, from (I.10), it is easy to see that $\tilde{\beta}_{T}^{\chi \omega(T)}$ is homogeneous of degree zero in the masses $m_{a}$. 
Applying recurrently the operator $\sum_{a=1}^{l} m_{a} \frac{\partial}{\partial m_{a}}$ upon the left hand side of (II.1) and using (II.1) itself for reduced subgraphs, we obtain

$$
\left(\sum_{a=1}^{l} m_{a} \partial / \partial m_{a}\right)^{r} I_{G}^{a s}(p, m)+(-)^{r-1} \sum_{\mathscr{F}_{r}} \chi_{\mathscr{F}_{r}} \prod_{T \in \mathscr{F}_{r}} 2 \tilde{\beta}_{[T / \operatorname{Max} T]_{\Lambda(T)}}^{\chi \omega(T)} I_{[G / \operatorname{Max} G]_{\Lambda(G)}}^{a s}(p, m)=0,
$$

where $\mathscr{F}_{r}$ is a forest or $r$ divergent generalized vertices, and $\chi_{\mathscr{F}_{r}}$ is a combinatorial factor similar to (I.8). If $r$ is chosen to be the largest number of divergent generalized vertices which can be organized in a forest, then $[G / \mathrm{Max} G]_{\Lambda(G)}$ is a convergent subgraph and $I_{[G / \operatorname{Max} G]}^{a s}(p, m)$ is homogeneous of degree zero in the masses $m_{a}$,

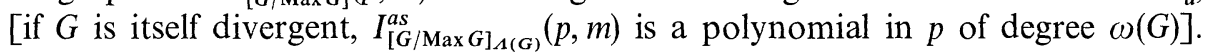

It is now possible to integrate back equation (II.2). We scale the masses $m_{a}$ by a factor $\lambda^{-1}$ so that the differential operator $\sum_{a=1}^{l} m_{a} \partial / \partial m_{a}$ acting upon Feynman amplitudes becomes $-\lambda \partial / \partial \lambda$. At each integration, the arbitrary constant is fixed at $\lambda=1$. We get

$$
I_{G}^{a s}(p, m / \lambda)=\sum_{\mathscr{F}} \chi_{\mathscr{F}} q(\mathscr{F}) !^{-1} \log ^{q(\mathscr{F})} \lambda \prod_{i=1}^{q(\mathscr{F})} 2 \tilde{\beta}_{\left[T_{i} / \operatorname{Max} T_{i}\right]_{\Lambda\left(T_{i}\right)}^{\chi \omega\left(T_{i}\right)}}^{I_{[G / \operatorname{Max} G]_{\Lambda(G)}}^{a s}}(p, m)
$$

In (II.3), the sum runs over all forests $\mathscr{F}$ of $q(\mathscr{F})$ divergent generalized vertices including the empty one. The above equation expresses the variation of the asymptotic part of a renormalized Feynman amplitude when its masses are scaled, in terms of the asymptotic parts corresponding to reduced subgraphs. The same result could be obtained by eliminating the functions $\phi$ in (I.7). Let us define a set of masses $\mu_{a}$ by

$$
m_{a}=\lambda \mu_{a}
$$

then

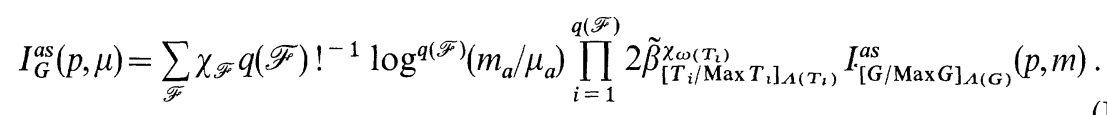

It turns out, that the right hand side of the above equation is independent of the masses $m_{a}$, and consequently the above combination of asymptotic parts has a limit when the masses $m_{a} \rightarrow 0$. In this limit the masses $\mu_{a}$ replace exactly the masses $m_{a}$ of the original asymptotic amplitude.

For a given Feynman graph, we define the following amplitude

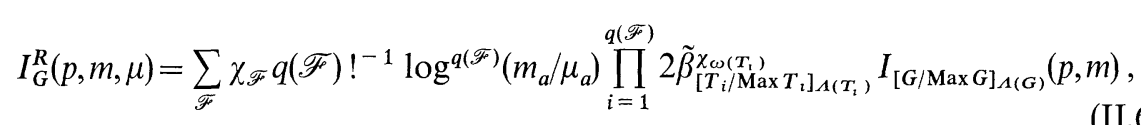

then

$$
I_{G}^{R}(p, m, \mu)=I_{G}^{a s}(p, \mu)+\Delta I_{G}^{R}(p, m, \mu),
$$

with

$$
\Delta I_{G}^{R}(p, m, \mu) \sim m \log ^{x} m .
$$




\section{Zero-Mass Limit of Lagrangian Field Theory}

We consider the following Lagrangian

$$
\mathscr{L}=\frac{1}{2} b(g) \partial_{\mu} \varphi \partial^{\mu} \varphi+\frac{1}{2} m^{2} a(g) \varphi^{2}-(c(g) / 4 !) \varphi^{4},
$$

defined as a formal power series in $g$. The functions $a(g), b(g)$, and $c(g)$ can be expanded as

$$
\begin{aligned}
& a(g)=1-\sum_{n=1}^{\infty} a_{n} g^{n}, \\
& b(g)=1-\sum_{n=1}^{\infty} b_{n} g^{n}, \\
& c(g)=g+\sum_{n=2}^{\infty} c_{n} g^{n} .
\end{aligned}
$$

From the Lagrangian (III.1), we can calculate in perturbation the $N$-points vertex functions $\Gamma_{(N)}(p, m, g)$ as infinite sums of Feynman amplitudes (I.3). We denote by $G_{N, n, i}$ the Feynman graph with $N$ external legs and $n$ 4-legs vertices, which enter in the calculation of $\Gamma_{(N)}(p, m, g)$ when $a(g)=b(g)=1$ and $c(g)=g ; i$ is a running index which takes values between 1 and $I(N, n)$ which is the number of such graphs. In Appendix A, we express $\Gamma_{(N)}(p, m, g)$ as a formal power series in $g$, taking into account the counterterm structure. It is convenient to decompose the coefficients $a_{n}, b_{n}$, and $c_{n}$ upon the graphs $G_{N, n, i}$ for $N=2$ and 4 ,

$$
\begin{aligned}
& a_{n}=\sum_{i=1}^{I(2, n)} \theta_{G_{2, n, i}} a_{G_{2, n, i}}, \\
& b_{n}=\sum_{i=1}^{I(2, n)} \theta_{G_{2, n, i}} b_{G_{2, n, i}}, \\
& c_{n}=\sum_{i=1}^{I(4, n)} \theta_{G_{4, n, i}} c_{G_{4, n, i}},
\end{aligned}
$$

where $\theta_{G_{N, n, i}}$ is a numerical constant which arises in Wick's contractions. Then,

$$
\begin{aligned}
& \Gamma_{(N)}(p, m, g)=\sum_{\gamma=0}^{\infty} g^{\gamma} \sum_{i=1}^{I(N, \gamma)} \theta_{G_{N, \gamma, i}} \\
& \cdot\left\{\sum_{\left\{R_{1}, \ldots, R_{t}\right\}} \prod_{\substack{R_{j} \\
\omega\left(R_{j}\right)=0}} c_{R_{j}} \prod_{\substack{R_{j} \\
\left(R_{j}\right)=2}} \mathcal{O}_{R_{j}} I\left[G_{N, \gamma, i} / \bigcup_{j=1}^{t} R_{j}\right](p, m)\right\} .
\end{aligned}
$$

In (III.4), $\left\{R_{1}, \ldots, R_{t}\right)$ is a (possibly empty) set of disjoint, divergent generalized vertices of $G_{N, \gamma, i} ; \mathcal{O}_{R_{j}}$ means that $\left(b_{R_{j}} k^{2}+a_{R_{j}} m^{2} \|\right)$ is introduced at the numerator of the Feynman integrand of the reduced graph $\left[G_{N, \gamma, i} / \bigcup_{j=1}^{t} R_{j}\right]$, for each quadratically divergent generalized vertex $R_{j}$ with external momenta $k\left(m^{2} \|\right.$ indicates an oversubtraction by 2 for the mass term); $I_{G}(p, m)$ is the renormalized Feynman amplitude (I.3) for the graph G. Equation (III.4) is a convenient way of writing the vertex functions $\Gamma_{(N)}(p, m, g)$ from which, in Appendix A we solve Bogolubov 
and Parasiuk [7], and Hepp [8] recurrence. At this point we do not impose any subtraction conditions upon $\Gamma_{(2)}$ and $\Gamma_{(4)}$; we let $\Gamma_{(N)}(p, m, g)$ to be a functional of the counterterm coefficients.

If we let the mass $m$ tends to zero in (III.4), we know from Section I that if

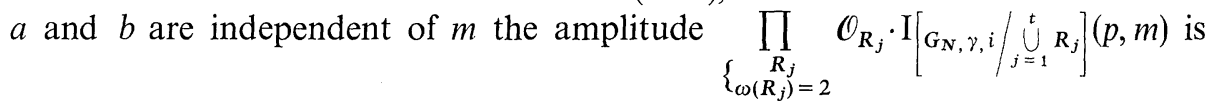
logarithmically divergent at non exceptional momentum. The behaviour of the vertex functions $\Gamma_{(N)}(p, m, g)$ when $m$ tends to zero depends greatly of the behaviour in $m$ of the counterterms. In this section, we find a choice of infrared logarithmically divergent counterterms such that $\Gamma_{(N)}(p, 0, g)$ exists at non exceptional momentum; with this choice, $\Gamma_{(N)}(p, m, g)$ is easily expressed in terms of $I_{G}^{R}(p, m, \mu)$ defined in (II.6). Let us calculate from (III.4) the quantity $m \partial \Gamma_{(N)}(p, m, g) / \partial m$. Three kinds of terms appear in the derivation $m(\partial / \partial m)$, namely $m \partial C_{R} / \partial m, m \partial \Theta_{R} / \partial m$, and $m \partial I_{G} / \partial m$. The last term can be transformed using Zimmermann's identity (I.9) (see Appendix A); we get

$$
\begin{aligned}
& m\left(\partial \Gamma_{(N)}(p, m, g) / \partial m\right)=2 \tilde{\Gamma}_{(N)}(p, m, g)+\sum_{\gamma=0}^{\infty} g^{\gamma} \sum_{i=1}^{I(N, \gamma)} \theta_{G_{N, \gamma, i}}
\end{aligned}
$$

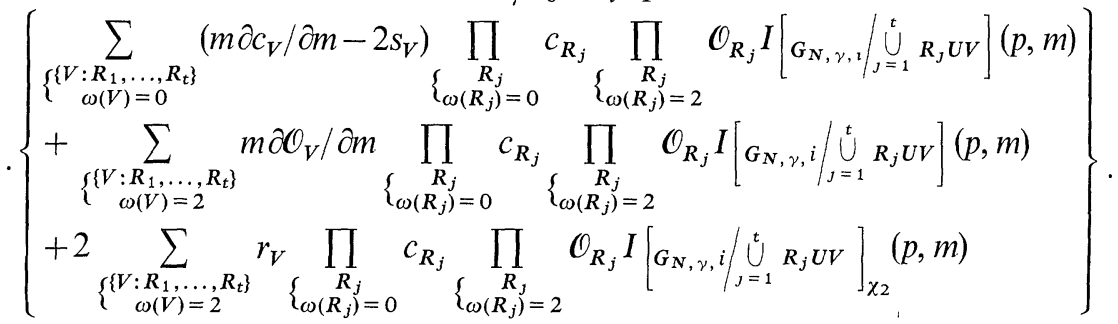

In (III.5), the quantities $\tilde{\Gamma}_{(N)}(p, m, g), r_{V}$ and $s_{V}$ are defined in Appendix A, respectively in (A.25), (A.34), and (A.35). The function $\tilde{\Gamma}_{(N)}(p, m, g)$ does have a zero-mass limit equal to zero if the counterterm coefficients $a_{G}, b_{G}$, and $c_{G}$ are at most logarithmically divergent when $m \rightarrow 0$. Consequently, if we can solve the system

$$
\begin{aligned}
& m \partial a_{V} / \partial m+2 a_{V}=0 \\
& m \partial b_{V} / \partial m+2 r_{V}=0 \\
& m \partial c_{V} / \partial m-2 s_{V}=0
\end{aligned}
$$

the curley bracket \{\} in (III.5) vanishes and then,

$$
\lim m \rightarrow 0\left\{m \partial \Gamma_{(N)}(p, m, g) / \partial m\right\}=0 .
$$

If we solve the system of differential Equations (III.6), we have found a set of functions $a(g), b(g)$, and $c(g)$ such that the vertex functions $\Gamma_{(N)}(p, m, g)$ derived from the corresponding Lagrangien (III.1) have a zero-mass limit. For such a system, we have

$$
m \partial \Gamma_{(N)}(p, m, g) / \partial m=2 \tilde{\Gamma}_{(N)}(p, m, g),
$$


as it can be seen directly using Zimmermann's identity for vertex functions; we define the functions $r(g)$ and $s(g)$ by

$$
\begin{aligned}
r(g) & =\sum_{n=2}^{\infty} r_{n} g^{n}, \\
r_{n} & =\sum_{i=1}^{I(2, n)} \theta_{G_{2, n, i}} r_{G_{2, n, i}},
\end{aligned}
$$

and similarly

$$
\begin{aligned}
s(g) & =\sum_{n=2}^{\infty} s_{n} g^{n}, \\
S_{n} & =\sum_{i=1}^{I(4, n)} \theta_{G_{4, n, i}} S_{G_{4, n, l}} .
\end{aligned}
$$

Then, we know that

$$
\begin{aligned}
\frac{m^{2}}{2} & N_{2}\left[\varphi^{2}\right] \Gamma_{(N)}(p, m, g) \\
& =\left\{\frac{m^{2}}{2} N_{4}\left[\varphi^{2}\right]+\frac{r(g)}{2} N_{4}\left[\partial_{\mu} \varphi \partial^{N} \varphi\right]-(s(g) / 4 !) N_{4}\left[\varphi^{4}\right]\right\} \Gamma_{(N)}(p, m, g),
\end{aligned}
$$

where we use Lowenstein's differential vertex operator [3].

Using Schwinger action principle and Equations (III.6) which become

$$
\begin{aligned}
& m \partial a(g) / \partial m+2[a(g)-1]=0 \\
& m \partial b(g) / \partial m-2 r(g)=0, \\
& m \partial c(g) / \partial m-2 s(g)=0
\end{aligned}
$$

we get Equation (III.8) since $\tilde{\Gamma}_{N}(p, m, g)$ is related to the soft mass insertion operator by (A.24).

The solution of the system of differential Equations (III.6) can be performed easily using (A.34) and (A.35) with the following boundary conditions

$$
a_{V}(m=\mu)=b_{V}(m=\mu)=c_{V}(m=\mu)=0,
$$

where $\mu$ is a given mass parameter. Then, we get

$$
a(g)=1,
$$

and

$$
b_{V}=\sum_{\mathscr{F}} \chi_{\mathscr{F}} q(\mathscr{F}) !^{-1} \log ^{q(\mathscr{F})}(m / \mu) \prod_{\mathscr{P} \in \mathscr{F}} 2 \tilde{\beta}_{[\mathscr{Y} / \mathbf{M} \text { ax } \mathscr{S}]_{\Lambda}(\mathscr{\varphi})}^{\chi_{\omega}(\mathscr{S})}
$$

where we sum over all forest $\mathscr{F}$ of $q(\mathscr{F})$ divergent generalized vertices containing the quadratically divergent graph $V$ itself. A similar equation holds for $c_{V}$ where $v$ is a logarithmically divergent graph. If we report these results for $a_{V}, b_{V}$, and $c_{V}$ 
in (III.4), we obtain

$$
\Gamma_{(N)}(p, m, \mu, g)=\sum_{\gamma=0}^{\infty} g^{\gamma} \sum_{i=1}^{I(N, \gamma)} \theta_{G_{N, \gamma, i}} I_{G_{N, \gamma, i}}^{R}(p, m, \mu),
$$

where $I_{G}^{R}(p, m, \mu)$ is defined in (II.6) and has a zero mass $m$ limit. Let us finally mention that the 2-points vertex function defined in (III.15) with zero-mass $m$, vanishes at $p^{2}=0$.

$$
\Gamma_{(2)}\left(p^{2}=0, m=0, \mu, g\right)=0,
$$

but the quantities $\left.\frac{d}{d p^{2}} \Gamma_{(2)}\left(p^{2}, m, \mu, g\right)\right|_{p^{2}=0}$ and $\Gamma_{(4)}\left(P_{i}=0, m, \mu, g\right)$ are infinite

sums of terms, each of them being logarithmically divergent when $m \rightarrow 0$.

\section{A New Subtraction Operator}

At the end of Section III, we are in a situation analog to the treatment which was given historically to the ultraviolet divergences. To avoid these divergences, it was convenient to regularize the Feynman amplitudes with a cut off; then introducing counterterms which were ultraviolet divergent when the cut-off is removed, it was possible to define ultraviolet convergent vertex functions $\Gamma_{(N)}(p, m, g)$. After Zimmermann's [11] work, we know that a renormalized Feynman amplitude can be defined in a compact form graph by graph, by-passing completely the introduction of any cut-off. Such a renormalized amplitude can also be defined in the Schwinger $\alpha$-parameters $[10,13]$ as given in (I.3). To treat the infrared divergences, we start from a massive theory and we find a set of counterterms which are logarithmically divergent when $m \rightarrow 0$ in such a way that the vertex functions have a zero-mass limit; this is achieved in last section. In Section IV, we want to describe a new subtraction scheme which defines in a compact form, graph by graph, an ultraviolet and infrared convergent amplitude at non exceptional momentum. This new subtraction scheme which can already be defined for a massive theory has all the properties of the usual subtraction scheme: it satisfies Bogolubov and Parasiuk [7], and Hepp [8] recurrence and it ensures the absolute convergence of the Feynman integrals; in addition it has the property to define an amplitude which has a zero-mass limit at non-exceptional momenta, for a graph satisfying (I.1). We define

$$
\begin{aligned}
I_{G}^{R}(p, m, \mu)= & \int_{0}^{\infty} \prod_{a=1}^{l} d \alpha_{a} e^{-\sum_{a=1}^{l} \alpha_{a} \mu_{a}^{2}} \\
& \cdot R\left\{e^{-\sum_{a=1}^{l} \alpha_{a}\left(m_{a}^{2}-\mu_{a}^{2}\right)} R^{-}\left\{\pi\left(\frac{-1}{\sqrt{\alpha_{a}}} \frac{\partial}{\partial z_{a}}\right) Z_{G}(\alpha, p, z)\right\}_{z_{a}=0}\right\} .
\end{aligned}
$$

The above amplitude has to be compared with the usual renormalized Feynman amplitude (I.3). We call this amplitude $I_{G}^{R}(p, m, \mu)$ because it is equal to the amplitude defined in (II.6) as it is proved below. A direct proof of absolute con- 
vergence and the fact that it satisfies Bogolubov, Parasiuk, and Hepp recurrence are shown somewhereelse [14] but the identity of (IV.1) and (II.6) already proves these two properties as well as the existence of the zero masses $m_{a}$ limit. In Equation (IV.1) the ratio $m_{a} / \mu_{a}$ is a constant for all lines $a$; the operator $R$ is the same as in (I.3) and in Ref. [10 and 13], and can be written under the form

$$
R=\prod_{\mathscr{S} \subseteq G}\left(1-\tau_{\mathscr{S}}^{-2 l(\mathscr{S})}\right)
$$

where the $\tau$ operators are generalized Taylor operators [10,13] and the product runs in any order over the $\left(2^{l}-1\right)$ subgraphs of $G$. The operator $R^{-}$is defined as

$$
R^{-}=\prod_{\mathscr{S} \subseteq G}\left(1-\tau_{\mathscr{S}}^{-2 l(\mathscr{\mathscr { S }})-1}\right) .
$$

To resume what $R$ and $R^{-}$does, we consider for instance a $\varphi^{4}$-type integrand; then let us simply say that $R^{-}$presubtract the quadratically divergent subgraphs at zero external momentum (only one subtraction) while $R$ subtracts the divergent subgraphs $\mathscr{S}(\omega(\mathscr{S})+1$ subtractions) at zero external momentum and at $m_{a}^{2}=\mu_{a}^{2}$.

Another way of writing $I_{G}^{R}(p, m, \mu)$ is

$$
\begin{aligned}
I_{G}^{R}(p, m, \mu)= & \int_{0}^{\infty} \prod_{a=1}^{l} d \alpha_{a} e^{-\sum_{a=1}^{l} \alpha_{a} \mu_{a}^{2}} \prod_{\mathscr{S} \subseteq G}\left(1-\Delta_{\mathscr{S}}^{-2 l(\mathscr{S})}\right) \\
& \cdot\left\{e^{-\sum_{a=1}^{l} \alpha_{a}\left(m_{a}^{2}-\mu_{a}^{2}\right)} \pi\left(\frac{-1}{\sqrt{\alpha_{a}^{\prime}}} \frac{\partial}{\partial z_{a}}\right) Z_{G}\left(\alpha^{\prime}, p, z\right)\right\}_{\substack{z_{a}=0 \\
\alpha_{a}^{\prime}=\alpha_{a}}} .
\end{aligned}
$$

In (IV.4),

$$
\Delta_{\mathscr{S}}^{n}=\tau_{\mathscr{S}}^{n}\left(\alpha, \alpha^{\prime}\right)+\tau_{\mathscr{S}}^{n-1}\left(\alpha^{\prime}\right)-\tau_{\mathscr{S}}^{n}\left(\alpha, \alpha^{\prime}\right) \tau_{\mathscr{S}}^{n-1}\left(\alpha^{\prime}\right)
$$

so that

$$
\left(1-\Delta_{\mathscr{S}}^{n}\right)=\left[1-\tau_{\mathscr{S}}^{n}\left(\alpha, \alpha^{\prime}\right)\right]\left[1-\tau_{\mathscr{S}}^{n-1}\left(\alpha^{\prime}\right)\right] .
$$

The generalized Taylor operator $\tau_{\mathscr{S}}^{n}\left(\alpha, \alpha^{\prime}\right)$ acting upon $\mathscr{Z}\left(\alpha, \alpha^{\prime}\right)$ means

$$
\left[\tau_{\varrho}^{n} \mathscr{Z}\left(\alpha, \alpha^{\prime}\right) \mid \begin{array}{l}
\alpha_{a} \rightarrow \varrho^{2} \alpha_{a}, a \in \mathscr{S} \\
\alpha_{a}^{\prime} \rightarrow \varrho^{2} \alpha_{a}^{\prime}, a \in \mathscr{S}
\end{array}\right]_{\varrho=1} \text { and the operator } \tau_{\mathscr{S}}^{n}\left(\alpha^{\prime}\right) \text { is the usual generalized }
$$

Taylor operator defined in Ref. [10] and [13]. The operators $\tau_{\mathscr{y}}^{n}\left(\alpha, \alpha^{\prime}\right)$ and $\tau_{\mathscr{\varphi}}^{n^{\prime}}\left(\alpha^{\prime}\right)$ commute. The proof of the equality between (IV.1) and (IV.4) is based upon the fact that the product $\prod_{\mathscr{S} \subseteq G}\left(1-\Delta_{\mathscr{\mathscr { S }}}^{-2 l(\mathscr{S})}\right)$ can be taken in any order as it is proved in Appendix B. We also prove in Appendix B the forest formula of diverging generalized vertices

$$
\prod_{\mathscr{S} \subseteq G}\left(1-\Delta_{\mathscr{S}}^{-2 l(\mathscr{S})}\right)\{\}=\left[1+\sum_{\mathscr{F}} \prod_{\mathscr{S} \in \mathscr{F}}\left(-\Delta_{\mathscr{T}}^{-2 l(\mathscr{S})}\right)\right]\{\},
$$

where the curly bracket \{\} is the same as the one in (IV.4). The forest formula of diverging generalized vertices is needed to prove directly Bogolubov, Parasiuk, and Hepp recurrence (see Ref. [13]). 
Let us now show the identity between (IV.4) and (II.6). We calculate

$$
\begin{aligned}
& \sum_{a=1}^{l} \mu_{a} \partial I_{G}^{R}(p, m, \mu) / \partial \mu_{a}=-2 \int_{0}^{\infty} \prod_{a=1}^{l} d \alpha_{a} e^{-\sum_{a=1}^{l} \alpha_{a} \mu_{a}^{2}} \\
& \cdot\left\{\left[\sum_{a=1}^{l} \alpha_{a} \mu_{a}^{2} \cdot \sum_{\mathscr{F}} \prod_{\mathscr{S} \in \mathscr{F}}(-\Delta)_{\mathscr{S}}^{-2 l(\mathscr{S})}\right)-\sum_{\mathscr{F}} \prod_{\mathscr{S} \in \mathscr{F}}\left(-\Delta_{\mathscr{S}}^{-2 l(\mathscr{S})}\right) \cdot \sum_{a=1}^{l} \alpha_{a} \mu_{a}^{2}\right] \\
& \left.\cdot e^{-\sum_{a=1}^{l} \alpha_{a}\left(m_{a}^{2}-\mu_{a}^{2}\right)} \pi\left(\frac{-1}{\sqrt{\alpha_{a}^{\prime}}} \frac{\partial}{\partial z_{a}}\right) Z_{G}\left(\alpha^{\prime}, p, z\right)\right\}_{\substack{z_{a}=0 \\
\alpha_{a}=\alpha_{a}^{\prime}}} .
\end{aligned}
$$

The technique to compute the curly bracket \{\} is similar to the technique used to prove Zimmermann's identity (I.9) and is exposed in Ref. [5] and [14] we obtain,

$$
\sum_{a=1}^{l} \mu_{a} \partial I_{G}^{R}(p, m, \mu) / \partial \mu_{a}=-2 \sum_{T} \tilde{\beta}_{T}^{\chi_{\omega(T)}} I_{[G / T]_{\chi_{\omega(T)}}^{R}}(p, m, \mu),
$$

where we sum over all divergent generalized vertices $T$. Such a differential equation has already been encountered in Section II. With, for boundary conditions $I_{G}^{R}(p, m, m)$ equal to $I_{G}(p, m)$, we get back Equation (II.6).

In this section, we have defined a family of subtraction operator depending upon the non-zero parameters $\mu_{a}$. The usual subtraction operator is obtained when $\mu_{a}=m_{a}$. The amplitudes obtained from such subtraction operators have a limit when the masses $m_{a} \rightarrow 0$ provided that the masses $\mu_{a}$ are $m_{a}$ independent.

\section{Conclusion}

In this paper we have defined a new subtraction procedure to renormalize Feynman amplitudes in perturbation theory. This procedure ensures the infrared and ultraviolet absolute convergence of all Feynman graphs at non exceptional momenta if at each vertex the degree of the coupling is larger or equal to four (boson line $+3 / 2$ fermion line + derivative coupling $\geqq 4$ ). It also satisfies Bogolubov, Parasiuk, and Hepp recurrence. Given, for instance, a $\varphi^{4}$ type Lagrangian

$$
\mathscr{L}(g)=\frac{1}{2} b(g) \partial_{\mu} \varphi \partial^{\mu} \varphi+\frac{1}{2} m^{2} a(g) \varphi^{2}-(c(g) / 4 !) \varphi^{4},
$$

we can built the corresponding vertex functions

$$
\begin{aligned}
& \Gamma_{(N)}(p, m, \mu, g)=\sum_{\gamma=0}^{\infty} g^{\gamma} \sum_{i=1}^{I(N, \gamma)} \theta_{G_{N, \gamma, i}}
\end{aligned}
$$

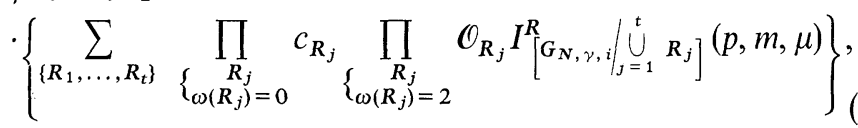

where the notations are defined in Section III and the Feynman amplitude $I_{G}^{R}(p, m, \mu)$ is given in (IV.1). If we impose to $\Gamma_{(2)}$ and $\Gamma_{(4)}$ the usual mass $m$-shell conditions, the $\mu$ dependence of $\Gamma_{(N)}$ disappear and we get back the usual vertex 
functions. On the other hand, if the counterterms are $m$ independent, the zeromass $m$ limit of $\Gamma_{(N)}$ obviously exists since $I_{G}^{R}(p, m, \mu)$ does have a zero-mass $m$ limit. If the counterterms are $m$ dependent, they must have a zero-mass $m$ limit if we want $\Gamma_{(N)}(p, 0, \mu, g)$ to exist. It is well known for instance, that with the usual mass $m$-shell conditions, $\Gamma_{(N)}(p, m, g)$ does not have a zero-mass $m$ limit but in the so-called $\mu$ renormalization where $\Gamma_{(2)}\left(p^{2}=m^{2}, m, g\right)$ is zero but the derivative $\frac{d}{d p^{2}} \Gamma_{(2)}$ and $\Gamma_{(4)}$ are subtracted in a $\mu$-point, $\Gamma_{(N)}(p, o, \mu, g)$ exists.

A similar subtraction procedure has been recently defined in momentum space by Lowenstein and Zimmermann [6] and we have no doubt that the amplitude (IV.1) is the $\alpha$-parameter version of their amplitude. The new Feynman amplitudes (IV.1) taken at masses $m_{a}$ equal to zero can be used to construct the so-called preasymptotic theory. It will be shown somewhere else that the amplitudes (IV.1) plays an important role in the analytic continuation in dimension $D$.

Acknowledgments. We wish to express our gratitude to Professor B. Schroer and to the Institut für Theoretische Physik for their kind hospitality.

\section{Appendix A. The Counterterms Structure in a $\varphi^{4}$ Type Theory}

\section{a) The Vertex Functions}

We consider the following Lagrangian defined as a formal power series in the parameter $g$.

$$
\mathscr{L}(\varphi, g)=\frac{1}{2} b(g) \partial_{\mu} \varphi \partial^{\mu} \varphi+\frac{1}{2} m^{2} a(g) \varphi^{2}-(c(g) / 4 !) \varphi^{4} .
$$

The functions $a(g), b(g)$, and $c(g)$ are formal power series in $g$

$$
\begin{aligned}
& a(g)=1-\sum_{n=1}^{\infty} a_{n} g^{n} \\
& b(g)=1-\sum_{n=1}^{\infty} b_{n} g^{n}, \\
& c(g)=g+\sum_{n=2}^{\infty} c_{n} g^{n} .
\end{aligned}
$$

The vertex functions $\Gamma_{(N)}(p, m, g)$ corresponding to the Lagrangian $\mathscr{L}(\varphi, g)$ where $N$ is the number of external legs, are infinite sums of Feynman amplitudes which may be constructed with the following Feynman rules: we associate to every vertex a function $c(g)$ and to every lines a propagator

$$
\pi(k, m, g)=\left[b(g) k^{2}+a(g) m^{2}\right]^{-1} .
$$

Let us label the one-line irreducible, connected graph which enter in the computation of $\Gamma_{(N)}(p, m, g)$ by $G_{N, n, i}$ where $n$ is the number of vertices and $i$ is a running index which take values from 1 up to $I(N, n)$ which is the number of such graphs in $\Gamma_{(N)}$. Then,

$$
\Gamma_{(N)}(p, m, g)=\sum_{n=0}^{\infty}[c(g)]^{n} \sum_{i=1}^{I(N, n)} \theta_{G_{N, n, i}} K_{G_{N, n, i}}(p, m, g),
$$


where $K_{G_{N, n, l}}(p, m, g)$ is the renormalized Feynman amplitude for the graph $G_{N, n, i}$ computed with the propagators (A.3), with subtraction at zero momentum, and $\theta_{G_{N, n, i}}$ is a numerical factor related to the symmetries of the graph $G_{N, n, i}$. Since we want to express $\Gamma_{(N)}$ as a formal power series in $g$, we first write the propagator (A.3) as

$$
\pi(k, m, g)=\left(k^{2}+m^{2}\right)^{-1} \sum_{q=0}^{\infty}\left\{\left([1-b(g)] k^{2}+[1-a(g)] m^{2} \|\right) /\left(k^{2}+m^{2}\right)\right\}^{q} .
$$

Each propagator (A.3) is then replaced by an infinite set of lines of the type

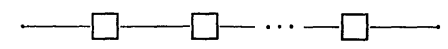

with $q$ box vertices $\left([1-b(g)] k^{2}+[1-a(g)] m^{2} \|\right)$, and $(q+1)$ propagators of the type $\left(k^{2}+m^{2}\right)^{-1}$. For the purpose of renormalization, we remind the reader that $m^{2} \|$ in the box vertex means an oversubtraction by two. In the above process, each graph $G_{N, n, i}$ generates an infinite number of new kind of graphs called $H_{N, n, i}\left(q_{1}, \ldots, q_{l}\right)$, where $q_{j}$ is the number of box vertices on the line $j$ corresponding to the line $j$ of the graph $G_{N, n, i}$. Equation (A.4) becomes

$$
\Gamma_{(N)}(p, m, g)=\sum_{n=0}^{\infty}[c(g)]^{n} \sum_{i=1}^{I(N, n)} \theta_{G_{N, n, l}} \sum_{\substack{q_{j}=0 \\ j=1, \ldots, l}}^{\infty} \prod_{j=1}^{l}\left[\mathcal{O}_{j}(g)\right]^{q_{j}} I_{H_{N, n, i}\left(q_{1}, \ldots, q_{l}\right)}(p, m),
$$

where the scalar amplitudes $I_{H_{N, n, i}\left(q_{1}, \ldots, q_{l}\right)}(p, m)$ are computed with the propagator $\left(k^{2}+m^{2}\right)^{-1}$. In (A.6), the operator $\mathcal{O}_{j}(g)$ acting upon $I_{H_{N, n, i}\left(q_{1}, \ldots, q_{l}\right)}(p, m)$ introduces in the Feynman integrand of the graph $H_{N, n, i}\left(q_{1}, \ldots, q_{l}\right)$ the numerator $\left([1-b(g)] k_{j}^{2}+[1-a(g)] m^{2} \|\right)$. Next, we use definitions (A.2) for the functions $a(g)$, $b(g)$, and $c(g)$. This is equivalent to the following: for each graph $H_{N, n, i}\left(q_{1}, \ldots, q_{l}\right)$, we define an infinite set of graphs such that at each (4-legs or box) vertex is attached a positive integer. Such graphs are denoted by

$$
F_{N, n, i}^{\gamma}\left(r_{1}, \ldots, r_{n} ; s_{11}, \ldots, s_{1 q_{1}} ; s_{21}, \ldots ; \ldots ; \ldots s_{l q_{l}}\right)
$$

or $F_{N, n, i}^{\gamma}(\{r\},\{s\})$ for simplicity. In this notation

$$
\gamma=\sum_{i=1}^{n} r_{i}+\sum_{i=1}^{l} \sum_{j=1}^{q_{i}} s_{i j}
$$

Then

$$
\begin{aligned}
\Gamma_{(N)}(p, m, g)= & \sum_{\gamma=0}^{\infty} g^{\gamma} \sum_{n=0}^{\gamma} \sum_{i=1}^{I(N, n)} \theta_{G_{N, n, i}} \\
& \cdot\left\{\sum_{\{q\},\{r\},\{s\}} \prod_{j=1}^{n} c_{r_{j}} \prod_{u=1}^{l} \prod_{v=1}^{q_{u}} \mathcal{O}_{S_{u v}} I_{H_{N, n, i}\left(q_{1}, \ldots, q_{l}\right)}(p, m)\right\},
\end{aligned}
$$

where the sums over $\{q\},\{r\}$, and $\{s\}$ are limited by the condition (A.7) and where the operator $\mathcal{O}_{s_{u v}}$ means that

$$
b_{s_{u v}} k_{u}^{2}+a_{s_{u v}} m^{2} \| \text {. }
$$


is introduced at the numerator of the Feynman integrand of the graph $H_{N, n, i}\left(q_{1}, \ldots, q_{l}\right)$. The formal power series (A.8) which defines the vertex functions $\Gamma_{(N)}$ can now be transformed by choosing the coefficients $a_{n}, b_{n}$, and $c_{n}$ in a way which suits the purpose of renormalization. We define

$$
\begin{aligned}
& a_{n}=\sum_{i=1}^{I(2, n)} \theta_{G_{2, n, i}} a_{G_{2, n, i}}, \\
& b_{n}=\sum_{i=1}^{I(2, n)} \theta_{G_{2, n, 2}} b_{G_{2, n, i}}, \\
& c_{n}=\sum_{i=1}^{I(4, n)} \theta_{G_{4, n, 2}} c_{G_{4, n, 2}} .
\end{aligned}
$$

The coefficients $a_{n}$ and $b_{n}$ are decomposed over each $n$ vertices, quadratically divergent, generalized vertex and the coefficients $c_{n}$ are decomposed over each $n$ vertices, logarithmically divergent generalized vertex.

If we replace $a_{n}, b_{n}$, and $c_{n}$ in Equations (A.8), (A.9) by their value from (A.10), then the graphs $F_{N, n, i}^{v}(\{r\},\{s\})$ can be considered as a reduced graph; indeed each box vertex with coefficients $a_{n}$ and $b_{n}$ can be replaced by a quadratically divergent generalized vertex with $n$ vertices, and each 4-legs vertex can be replaced by a logarithmically divergent generalized vertex with $n$ vertices. In this process, we generate a graph $G_{N, \gamma, j}$. The graph $F_{N, n, i}^{\gamma}\{\{r\},\{s\})$ is the reduced graph obtained from $G_{N, \gamma, j}$ by contracting into points the disjoint, logarithmically and quadratically divergent generalized vertices defined by $\{r\}$ and $\{s\}$. Conversely, given a graph $G_{N, \gamma, j}$, all possible set of disjoint, logarithmically or quadratically divergent generalized vertices, defined a possible graph $F_{N, n, i}^{\gamma}(\{r\},\{s\})$ which by the above process, generates back $G_{N, \gamma, j}$. Using the combinatoric relation

$$
\theta_{G}=\theta_{\left[\frac{G}{R}\right]} \theta_{R}
$$

between the numerical coefficients for a graph $G$, a subgraph $R$ and the reduced subgraph $\left[\frac{G}{R}\right]$, we can write $\Gamma_{(N)}$ as

$$
\begin{aligned}
\Gamma_{(N)}(p, m, g)= & \sum_{\gamma=0}^{\infty} g^{\gamma} \sum_{i=1}^{I(N, \gamma)} \theta_{G_{N, \gamma, i}} \\
& \cdot\left\{\sum_{\left\{R_{1}, \ldots, R_{t}\right\}} \prod_{\substack{R_{j} \\
\boldsymbol{R}_{\omega, j}=0}} c_{R_{j}} \prod_{\substack{R_{j} \\
\left\{\omega\left(R_{j}\right)=2\right.}} \mathcal{O}_{R_{j}} I_{\left[G_{N, \gamma, i} / \cup R_{j}\right]}(p, m)\right\},
\end{aligned}
$$

where $\left\{R_{1}, \ldots, R_{t}\right\}$ is a (possibly empty) set of disjoint, divergent, generalized vertices of $G_{N, \gamma, i}$, and where the operator $\mathcal{O}_{R_{j}}$ means that $\left(b_{R_{j}} k^{2}+a_{R_{j}} m^{2} \|\right)$ is introduced at the numerator of the Feynman integrand of the graph $\left[G_{N, \gamma, i} / \bigcup_{j=1}^{t} R_{j}\right]$ for each quadratically divergent generalized vertex $R_{j}$ with external momentum $k$. Let us remind here that the counterterm structure given by (A.2), (A.10) is such that the vertex functions $\Gamma_{(N)}$ can be written in the form (A.12), which is nothing but the starting point of Bogolubov and Parasiuk [7], and Hepp [8] recurrence. 
As an application of the form (A.12), let us solve for the coefficients $a_{R}, b_{R}$, and $c_{R}$ when we impose the usual mass-shell conditions

$$
\left\{\begin{array}{l}
\Gamma_{(2)}\left(p^{2}=-m^{2}, m, g\right)=0, \\
\left.\frac{d \Gamma_{(2)}}{d p^{2}}\left(p^{2}, m, g\right)\right|_{p^{2}=-m^{2}}=1, \\
\Gamma_{(4)}\left(p_{i} \cdot p_{j}=\frac{m^{2}}{3}\left(1-4 \delta_{i j}\right), m, g\right)=g .
\end{array}\right.
$$

Equations (A.13.a, b) implies for each graph $G_{2, \gamma, i}$

$$
\begin{aligned}
& \sum_{\left\{\boldsymbol{R}_{1}, \ldots, \boldsymbol{R}_{t}\right\}} \prod_{\substack{\boldsymbol{R}_{j} \\
\omega\left(\boldsymbol{R}_{j}\right)=0}} c_{\boldsymbol{R}_{j}} \prod_{\substack{\boldsymbol{R}_{j} \\
\omega\left(\boldsymbol{R}_{j}\right)=2}} \mathcal{O}_{\boldsymbol{R}_{j}} I\left[G_{N, \gamma, i} / \bigcup_{j}^{t} R_{j}\right]\left(p^{2}=-m^{2}, m\right)=0,
\end{aligned}
$$

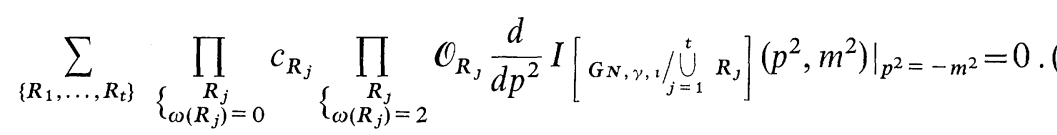

If we specify out in (A.14), the set of disjoint subgraphs equal to the graph $\left\{G_{2, \gamma, i}\right\}$ itself, then using

$$
\mathcal{O}_{G_{2, \gamma, i}} I_{\left[G_{2, \gamma, i} / G_{2, \gamma, i}\right)}(p, m)=b_{G_{2, \gamma, i}} p^{2}+a_{G_{2, \gamma, i}} m^{2},
$$

we obtain

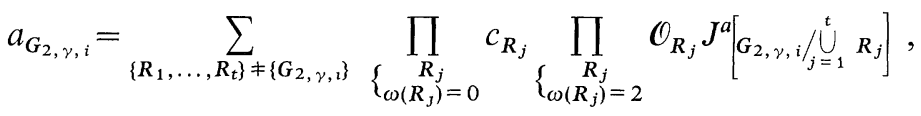

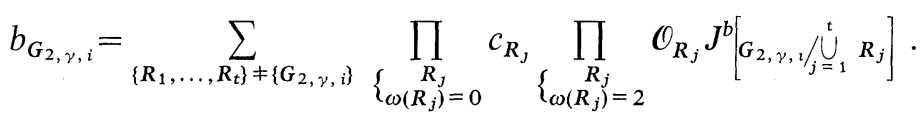

Similarly, from (A.13.c), we obtain for each graph $G_{4, \gamma, i}$

$$
c_{G_{4, \gamma, l}}=\sum_{\left\{R_{1}, \ldots, R_{t}\right\} \neq\left\{G_{4, \gamma, 2}\right\}} \prod_{\substack{\boldsymbol{R}_{j} \\ \omega\left(R_{j}\right)=0}} c_{R_{j}} \prod_{\substack{\boldsymbol{R}_{j} \\ \omega\left(\boldsymbol{R}_{j}\right)=2}} \mathcal{O}_{\boldsymbol{R}_{j}} J^{c}\left[G_{4, \gamma, i} / \bigcup_{j=1}^{t} R_{j}\right] .
$$

In (A.16), for a given graph $G=\left[G_{N, \gamma, i} / \bigcup_{j=1}^{t}\right], \quad(N=2,4)$,

$$
\begin{aligned}
& J_{G}^{a}=-\frac{1}{m^{2}}\left[I_{G}\left(p^{2}=-m^{2}, m\right)+\left.m^{2} \frac{d}{d p^{2}} I_{G}\left(p^{2}, m\right)\right|_{p^{2}=-m^{2}}\right] \\
& J_{G}^{b}=-\left.\frac{d}{d p^{2}} I_{G}\left(p^{2}, m\right)\right|_{p^{2}=-m^{2}} \\
& J_{G}^{c}=-I_{G}\left(p_{i} \cdot p_{j}=\frac{m^{2}}{3}\left(1-4 \delta_{i j}\right), m\right) .
\end{aligned}
$$

Equations (A.16) can be solved by recurrence; we find

$$
a_{G_{2, \gamma, i}}=\sum_{\mathscr{F},} \prod_{\substack{R \in \mathscr{F}^{\prime} \\ \omega(R)=2}} J_{[R / \operatorname{Max} R]}^{\mathscr{O}} \prod_{\substack{R \in \mathscr{F}^{\prime} \\ \omega(R)=0}} J_{[R / \operatorname{Max} R]}^{c} J_{\left[G_{2, \gamma, i} / \operatorname{Max} G_{2, \gamma, i}\right]}^{a},
$$


and two similar equations for $b_{G_{2, \gamma, 2},}$ and $C_{G_{4, \gamma, i}}$, with $J^{a}$ replaced by $J^{b}$ or $J^{c}$. In (A.18), we sum over all forests $\mathscr{F}^{\prime}$ (including the empty one) of divergent generalized vertices different from $G_{2, \gamma, i}$; the symbol $[R / \operatorname{Max} R]$ is defined in the introduction; the operator $\boldsymbol{J}_{R}^{0}$ means that $\left(J_{R}^{b} k^{2}+J_{R}^{a} m^{2} \|\right)$ is introduced at the numerator of the Feynman integrand of the graph $R^{\prime}$ where $R^{\prime} \in \mathscr{F}^{\prime}$ and $R \subset R^{\prime}$ and is maximal in $R^{\prime}$. The operator notation used in (A.18) necessitates that if $R \subset R^{\prime}, \boldsymbol{J}_{R}^{\mathfrak{o}}$ is written at the left of $\boldsymbol{J}_{R^{\prime}}^{\mathfrak{0}}$.

If we report the counterterms $a_{G_{2, \gamma, i}}, b_{G_{2, \gamma, i}}$, and $C_{G_{4, \gamma, i}}$ in Equation (A.12), we find

$$
\begin{aligned}
\Gamma_{(N)}(p, m, g)= & \sum_{\gamma=0}^{\infty} g^{\gamma} \sum_{i=1}^{I(N, \gamma)} \theta_{G_{N, \gamma, i}} \\
& \cdot\left\{\sum_{\mathscr{F}} \prod_{\substack{R \in \mathscr{F} \\
(R)=2}} J_{[R / \operatorname{Max} R]}^{\left.\mathscr{O}(R)=\prod_{\substack{R \in \mathscr{F} \\
\omega(R)=0}} J_{[R / \operatorname{Max} R]}^{c} I_{\left[G_{N}, \gamma, i\right.} / \operatorname{Max} G_{N, \gamma, i}\right]}(p, m)\right\},
\end{aligned}
$$

where now we sum over all forests $\mathscr{F}$ (including the empty one) of the graph $G_{N, \gamma, i}$.

\section{b) Vertex Insertions}

We define after Lowenstein [3] the three following vertex insertions

$$
\begin{aligned}
-\frac{m^{2}}{2} N_{4}\left[\varphi^{2}\right] \cdot \Gamma_{(N)}(p, m, g) & =\partial \Gamma_{(N)}(p, m, g) / \partial a(g), \\
-\frac{1}{2} N_{4}\left[\partial_{\mu} \varphi \partial^{\mu} \varphi\right] \cdot \Gamma_{(N)}(p, m, g) & =\partial \Gamma_{(N)}(p, m, g) / \partial b(g), \\
(1 / 4 !) N_{4}\left[\varphi^{4}\right] \cdot \Gamma_{(N)}(p, m, g) & =\partial \Gamma_{(N)}(p, m, g) / \partial c(g) .
\end{aligned}
$$

From (A.4), we obtain

$$
\begin{aligned}
& \partial \Gamma_{(N)}(p, m, g) / \partial a(g)=\sum_{n=0}^{\infty}[c(g)]^{n} \sum_{i=1}^{I(N, n)} \theta_{G_{N, n, i}} \frac{m}{2} \frac{\partial}{\partial m} K_{G_{N, n, i}}(p, m, g), \\
& \partial \Gamma_{(N)}(p, m, g) / \partial b(g)=-\sum_{n=0}^{\infty}[c(g)]^{n} \sum_{i=1}^{I(N, n)} \theta_{G_{N, n, i}} \sum_{j=1}^{l} K_{\left[G_{N, n, i}\right] x_{j}}(p, m, g), \\
& \partial \Gamma_{(N)}(p, m, g) / \partial c(g)=\sum_{n=0}^{\infty} n[c(g)]^{n-1} \sum_{i=1}^{I(N, n)} \theta_{G_{N, n, i}} K_{G_{N, n, i}}(p, m, g) .
\end{aligned}
$$

In (A.21.b), $\left[G_{N, n, i}\right]_{\chi_{j}}$ is the graph $G_{N, n, i}$ where a vertex $k_{j}^{2}$ is inserted on the line $j$. Let us mention here the counting identity; using the topological relation $4 n-2 l=N$ for each graph $G_{N . n . i}$, we have

$$
\begin{aligned}
& 2 a(g) \partial \Gamma_{(N)}(p, m, g) / \partial a(g)+2 b(g) \partial \Gamma_{(N)}(p, m, g) / \partial b(g)+4 c(g) \partial \Gamma_{(N)}(p, m, g) / \partial c(g) \\
& =N \Gamma_{(N)}(p, m, g) .
\end{aligned}
$$


The same transformation from (A.4) to (A.12) can be performed upon the Equations (A.21); we get

$$
\begin{aligned}
& \partial \Gamma_{(N)}(p, m, g) / \partial a(g)=\sum_{\gamma=0}^{\infty} g^{\gamma} \sum_{i=1}^{I(N, \gamma)} \theta_{G_{N, \gamma, i}} \\
& \cdot\left\{\sum_{\left\{\boldsymbol{R}_{1}, \ldots, \boldsymbol{R}_{t}\right\}} \prod_{\substack{\boldsymbol{R}_{j} \\
\omega\left(\boldsymbol{R}_{j}\right)=0}} c_{\boldsymbol{R}_{j}} \prod_{\substack{\boldsymbol{R}_{j} \\
\omega\left(\boldsymbol{R}_{J}\right)=2}} \mathcal{O}_{\boldsymbol{R}_{j}} \cdot \frac{m}{2} \frac{\partial}{\partial m} I\left[G_{N, \gamma, i} / \bigcup_{J=1}^{t} \boldsymbol{R}_{j}\right](p, m)\right\}, \\
& \partial \Gamma_{(N)}(p, m, g) / \partial b(g)=-\sum_{\gamma=0}^{\infty} g^{\gamma} \sum_{i=1}^{I(N, \gamma)} \theta_{G_{N, \gamma, i}}
\end{aligned}
$$

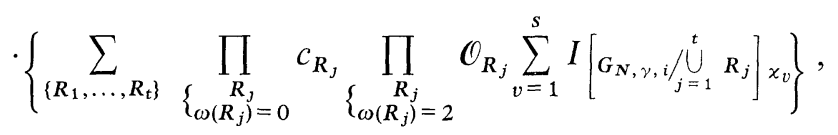

where $s$ is the total number of lines of $\left[G_{N, \gamma, i} / \bigcup_{j=1}^{t} R_{j}\right]$

$$
\begin{aligned}
& \partial \Gamma_{(N)}(p, m, g) / \partial c(g)=\sum_{\gamma=0}^{\infty} g^{\gamma-1} \sum_{i=1}^{I(N, \gamma)} \theta_{G_{N, \gamma, i}}
\end{aligned}
$$

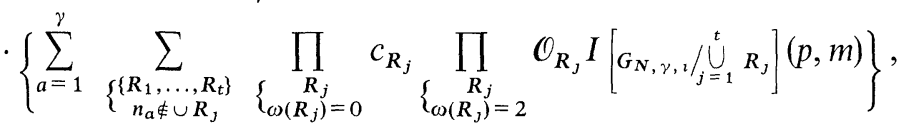

where we sum over all vertices $n_{a}$ of the graph $G_{N, \gamma, i}$. It is also useful to define the soft mass insertion

$$
\frac{-m^{2}}{2} N_{2}\left[\varphi^{2}\right] \cdot \Gamma_{(N)}(p, m, g)=\tilde{\Gamma}_{N}(p, m, g),
$$

where

$$
\begin{aligned}
& \tilde{\Gamma}_{N}(p, m, g)=-\sum_{\gamma=0}^{\infty} g^{\gamma} \sum_{i=1}^{I(N, \gamma)} \theta_{G_{N, \gamma, i}}
\end{aligned}
$$

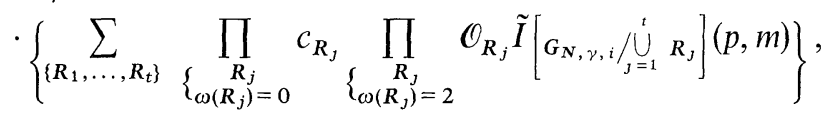

with $\tilde{I}_{G}$ defined in (I.9).

More generally, if

$$
\begin{aligned}
\phi(g) & =\sum_{n=2}^{\infty} \phi_{n} g^{n} \\
\phi_{n} & =\sum_{i=1}^{I(2, n)} \theta_{G_{2, n, i}} \phi_{G_{2, n, i}},
\end{aligned}
$$

then,

$$
\begin{aligned}
& \phi(g) \partial \Gamma_{(N)}(p, m, g) / \partial a(g)=-\sum_{\gamma=0}^{\infty} g^{\gamma} \sum_{i=1}^{I(N, \gamma)} \theta_{G_{N, \gamma, i}}
\end{aligned}
$$

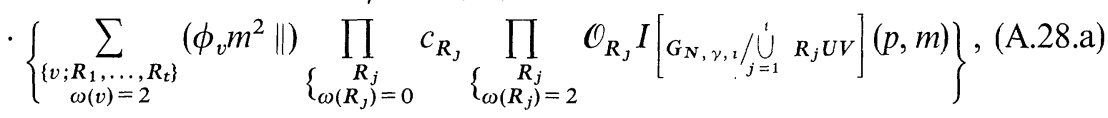


where $V$ is a quadratically divergent generalized vertex of $G_{N, \gamma, i}$. Similarly

$$
\begin{aligned}
& \phi(g) \partial \Gamma_{(N)}(p, m, g) / \partial b(g)=-\sum_{\gamma=0}^{\infty} g^{\gamma} \sum_{i=1}^{I(N, \gamma)} \theta_{G_{N, \gamma, i}}
\end{aligned}
$$

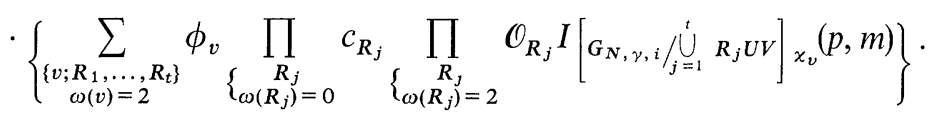

Also, if in (A.27), the sum runs over logarithmically divergent graphs, we get

$$
\begin{aligned}
& \phi(g) \partial \Gamma_{(N)}(p, m, g) / \partial c(g)=\sum_{\gamma=0}^{\infty} g^{\gamma} \sum_{i=1}^{I(N, \gamma)} \theta_{G_{N, \gamma, i}} \\
& \cdot\left\{\sum_{\substack{\left\{v ; R_{1}, \ldots, R_{t}\right\} \\
\omega(v)=0}} \phi_{v} \prod_{\substack{R_{j} \\
\omega\left(R_{j}\right)=0}} c_{R_{j}} \prod_{\left\{\begin{array}{c}
R_{j} \\
\omega\left(R_{j}\right)=2
\end{array}\right.} \mathcal{O}_{R_{j}} I\left[G_{N, \gamma, 2} / \bigcup_{j=1}^{t} R_{j} U V\right](p, m)\right\} .
\end{aligned}
$$

\section{c) Zimmermann's Identity}

In Section I, we already mention Zimmermann's identity for a Feynman amplitude. Here we intend to show the consequence of Equation (I.9) upon the vertex functions $\Gamma_{(N)}(p, m, g)$. From (A.23.a) and (A.25), we get

$$
\begin{aligned}
& \partial \Gamma_{(N)}(p, m, g) / \partial a(g)-\tilde{\Gamma}_{N}(p, m, g)=\sum_{\gamma=0}^{\infty} g^{\gamma} \sum_{i=1}^{I(N, \gamma)} \theta_{G_{N, \gamma, i}} \\
& \cdot\left[\sum_{\left\{R_{1}, \ldots, R_{t}\right\}} \prod_{\substack{R_{j} \\
\omega\left(R_{j}\right)=0}} c_{R_{j}} \prod_{\substack{R_{j} \\
\omega\left(R_{j}\right)=2}} \mathcal{O}_{R_{j}}\right. \\
& \text {. } \left.\left\{\frac{m}{2} \frac{\partial}{\partial m}\left[G_{N, \gamma, i} / \bigcup_{j=1}^{t} R_{j}\right](p, m)+\tilde{I}\left[G_{N, \gamma, i} \bigcup_{j=1}^{t} R_{J}\right](p, m)\right\}\right]
\end{aligned}
$$

The curly bracket \{\} can be transformed by using Equation (I.9),

$$
\begin{aligned}
& \partial \Gamma_{(N)}(p, m, g) / \partial a(g)-\tilde{\Gamma}_{N}(p, m, g)=-\sum_{\gamma=0}^{\infty} g^{\gamma} \sum_{i=1}^{I(N, \gamma)} \theta_{G_{N, \gamma, i}}
\end{aligned}
$$

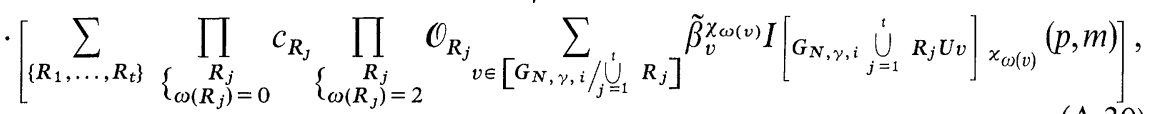

where $v$ is a divergent generalized vertex of $\left[G_{N, \gamma, i} / \bigcup_{j=1}^{t} R_{j}\right]$. We now interchange the summation over $v$ and the summation over the sets $\left\{R_{1}, \ldots, R_{t}\right\}$; let us partition the set $\left\{R_{1}, \ldots, R_{t}\right\}$ into two sets in regards to $v$ : the first set called $\{R\}_{1}$ is such that the contracted points $R_{k}$ in $\left[G_{N, \gamma, i} / \bigcup_{j=1}^{t} R_{j}\right]$ do not belong to $v$, and the second set $\{R\}_{2}$ is such that each contracted point $R_{k}$ belongs to $v$. We define in $G_{N, \gamma, i}$ the generalized vertex $v^{\prime}$ such that $v=\left[v^{\prime} / \bigcup_{R_{j} \in\{R\}_{2}} R_{j}\right]$ and we note that the superficial degree of divergence $\omega\left(v^{\prime}\right)=\omega(v)$ since each contraction of a quadratically divergent generalized vertex $R$ is associated to a derivate coupling of degree 2 
generated from the operator $\mathcal{O}_{R}$. Equation (A.30) can be rewritten as

$$
\begin{aligned}
& \partial \Gamma_{(N)}(p, m, g) / \partial a(g)-\tilde{\Gamma}_{(N)}(p, m, g)=-\sum_{\gamma=0}^{\infty} g^{\gamma} \sum_{i=1}^{I(N, \gamma)} \theta_{G_{N, \gamma, i}}
\end{aligned}
$$

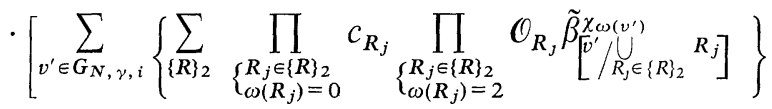

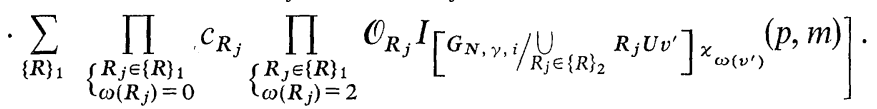

In (A.31), we sum over all (possibly empty) sets $\{R\}_{2}$ of disjoint, divergent, generalized vertices of $v^{\prime}$ ( $v^{\prime}$ itself excluded); the curly bracket \{\} in the above equation plays the same role as $\phi_{v}$ in Equation (A.28). We define the functions $r(g)$ and $s(g)$ as

$$
\begin{aligned}
r_{n} & =\sum_{i=1}^{I(2, n)} \theta_{G_{2, n, i}} r_{G_{2, n, i}} \text { for } n \geqq 2, \\
r(g) & =\sum_{n=2}^{\infty} r_{n} g^{n},
\end{aligned}
$$

and

$$
\begin{aligned}
s_{n} & =\sum_{i=1}^{I(4, n)} \theta_{G_{4, n, i}} s_{G_{4, n, i}} \text { for } n \geqq 2, \\
s(g) & =\sum_{n=2}^{\infty} s_{n} g^{n},
\end{aligned}
$$

where

$$
r_{G}=-\sum_{\left\{R_{1}, \ldots, R_{t}\right\} \neq\{G\}} \prod_{\substack{R_{j} \\
\omega\left(R_{j}\right)=0}} c_{R_{j}} \prod_{\left\{\begin{array}{c}
R_{j} \\
\omega\left(R_{j}\right)=2
\end{array}\right.} \mathcal{O}_{R_{j}} \tilde{\beta}_{\left[G / J_{j=1}^{t} R_{j}\right]}^{\chi_{2}},
$$

for a quadratically divergent graph $G$, and

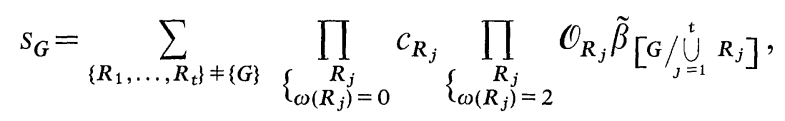

for a logarithmically divergent graph $G$. Then, a direct application of (A.28) gives

$$
\begin{aligned}
\tilde{\Gamma}_{N}(p, m, g)= & \partial \Gamma_{(N)}(p, m, g) / \partial a(g)+r(g) \partial \Gamma_{(N)}(p, m, g) / \partial b(g) \\
& +s(g) \partial \Gamma_{(N)}(p, m, g) / \partial c(g) .
\end{aligned}
$$

Equations (I.9) and (A.36) are due to Zimmermann [11].

\section{Appendix B. Different Forms of the New Subtraction Scheme}

The new subtraction scheme as written in Equation (IV.4), introduces the operator $\prod_{\mathscr{S} \cong G}\left(1-\Delta_{\mathscr{S}}^{-2 l(\mathscr{S})}\right)$ acting upon a function $\mathscr{Z}\left(\alpha, \alpha^{\prime}\right)$ which has the property of having a simultaneous Laurent series in the dilatation variables corresponding to sub- 
graphs which form a forest, independently of the fact that the variables $\alpha^{\prime}$ alone or the variables $\alpha$ and $\alpha^{\prime}$ are dilated. This property of the function $\mathscr{Z}\left(\alpha, \alpha^{\prime}\right)$ is extensively used in what follows. In this Appendix, we follow closely the Appendix B of Ref. [13] which proves the same kind of theorems for the usual subtraction scheme.

\section{The Nested Forest Formula}

\section{Theorem.}

$$
\prod_{\mathscr{S} \leqq G}\left(1-\Delta_{\mathscr{S}}^{-2 l(\mathscr{S})}\right) \mathscr{Z}\left(\alpha, \alpha^{\prime}\right)=\left[1+\sum_{\mathscr{N}} \prod_{\mathscr{S} \in \mathscr{N}}\left(-\Delta_{\mathscr{S}}^{-2 l(\mathscr{S})}\right)\right] \mathscr{Z}\left(\alpha, \alpha^{\prime}\right)
$$

where we sum over all sets of nested subgraphs. Consequently, the product in the left hand side of (B.1) is independent of the order of application.

Proof. We just mention here the main steps of this proof and the differences with the proof of Ref. [13]. Equation (B.1) is proved by recurrence. Each step of the recurrence is proved if we can show that, given a subgraph $\mathscr{S}_{n}$, and given a set $\mathscr{W}_{n-1}$ of $(n-1)$ subgraphs $\left\{\mathscr{S}_{1}, \ldots, \mathscr{S}_{n-1}\right\}$ such that at least one of them is either disjoint or overlapping with $\mathscr{S}_{n}$, we have

$$
\prod_{\substack{\mathscr{S} \neq \mathscr{S}_{n} \\ \mathscr{S}_{\mathscr{W}} \mathscr{W}_{n-1}}}\left(1-\Delta_{\mathscr{S}}^{-2 l(\mathscr{S})}\right)\left(-\Delta_{\mathscr{S}_{n}}^{-2 l\left(\mathscr{S}_{n}\right)}\right) \sum_{\mathscr{N} \in \mathscr{E}_{n-1}^{\prime}} \prod_{\mathscr{S} \in \mathscr{N}}\left(-\Delta_{\mathscr{S}}^{-2 l(\mathscr{S})}\right) \mathscr{Z}\left(\alpha, \alpha^{\prime}\right)=0 .
$$

In (B.2), $\mathscr{E}_{n-1}^{\prime}$ is the set of all forests of nested elements built with the subgraphs of $\mathscr{W}_{n-1}$ and which does contain at least one element either disjoint or overlapping with $\mathscr{S}_{n}$. To any nest $\mathscr{N} \in \mathscr{E}_{n-1}^{\prime}$, we define its $\mathscr{S}_{n}$-maximal nest $\mathscr{G}$. Then, the nest $\mathscr{G}$ can be decomposed into three subnests (see Ref. [13] for their definition)

$$
\mathscr{G}=\mathscr{B} \cup \mathscr{K} \cup \mathscr{H},
$$

where $\mathscr{B} \cup \mathscr{K}$ contains necessarily at least one element either disjoint or overlapping with $\mathscr{S}_{n}$. The elements of $\mathscr{B} \cup \mathscr{K}$ are elements of $\mathscr{W}_{n-1}$; on the other hand, the elements of $\mathscr{H}$ may not belong to $\mathscr{W}_{n-1}$. All nests $\mathscr{N}$ (which does not necessarily belong to $\mathscr{E}_{n-1}^{\prime}$ ) with the same $\mathscr{S}_{n}$-maximal nest $\mathscr{G}$ defines an equivalent class and we have

$$
\mathscr{N}=\mathscr{B} \cup \mathscr{K} \cup \mathscr{H}^{\prime} \quad \text { with } \quad \mathscr{H}^{\prime} \subseteq \mathscr{H} .
$$

When we sum over all nests which belong to the same equivalent class, we form the quantity

$$
\prod_{\substack{\mathscr{S} \neq \mathscr{S}_{n} \\ \mathscr{S}_{\mathscr{S}} \notin \mathscr{W}_{n-1}}}\left(1-\Delta_{\mathscr{S}}^{-2 l(\mathscr{S})}\right)\left(-\Delta_{\mathscr{S}_{n}}^{-2 l\left(\mathscr{S}_{n}\right)}\right) \prod_{\mathscr{S} \in \mathscr{B} \cup \mathscr{K}}\left(-\Delta_{\mathscr{S}}^{-2 l(\mathscr{S})}\right) \prod_{\mathscr{S} \in \mathscr{H}}\left(1-\Delta_{\mathscr{S}}^{-2 l(\mathscr{S})}\right) \mathscr{Z}\left(\alpha, \alpha^{\prime}\right) .
$$

If we use the property of the generalized Taylor operator (see Ref. [13], (A.9)) that

$$
\begin{aligned}
& \ldots\left(1-\Delta_{\mathscr{S}}^{-2 l(\mathscr{S})}\right) \ldots \Delta_{\mathscr{S}_{n}}^{-2 l\left(\mathscr{S}_{n}\right)} \ldots \mathscr{Z}\left(\alpha, \alpha^{\prime}\right) \\
& \quad=\ldots\left(1-\Delta_{\mathscr{S}}^{-2 l(\mathscr{S})}\right) \ldots \Delta_{\mathscr{S}_{n}}^{-2 l\left(\mathscr{S}_{n}\right)} \ldots\left(1-\Delta_{\mathscr{S}}^{-2 l(\mathscr{S})}\right) \ldots \mathscr{Z}\left(\alpha, \alpha^{\prime}\right),
\end{aligned}
$$


then, the sum of the expressions (B.5) over all equivalent classes is the left hand side of (B.2). The detailed proof that, for each equivalent class, the expression (B.5) is null, is too long to be reported here and we refer the reader to Ref. [14] for a more complete version. Let us simply say that in the function $\mathscr{Z}\left(\alpha, \alpha^{\prime}\right)$, we dilate the variables $\alpha_{a}^{\prime}$ and $\left(\alpha_{a}, \alpha_{a}^{\prime}\right)$ for $a \in B_{s} \in \mathscr{B}$ respectively by $\xi_{s}^{\prime 2}$ and $\xi_{s}^{2}$, for $a \in K_{j} \in \mathscr{K}$ by $\sigma_{j}^{\prime 2}$ and $\sigma_{j}^{2}$, for $a \in H_{j} \in \mathscr{H}$ by $\chi_{j}^{\prime 2}$ and $\chi_{j}^{2}$, for $a \in \mathscr{S}_{n}$ by $\beta^{\prime 2}$ and $\beta^{2}$. After application of the operators $\Delta$ relative to the elements of $\mathscr{K}$, and after using the Taylor remainder integral representation for the elements of $\mathscr{H}$, we obtain a sum of terms each of them containing in factor a term $\beta^{p} \beta^{\prime} p^{\prime}$, with

$$
p>-2 l\left(\mathscr{S}_{n}\right)
$$

and

$$
p^{\prime} \geqq-2 l\left(\mathscr{S}_{n}\right)
$$

The application of $\Delta_{\mathscr{S}_{n}}^{-2 l\left(\mathscr{S}_{n}\right)}$ over these terms gives zero.

\section{The Forest Formula}

\section{Theorem.}

$$
\prod_{\mathscr{S} \subseteq G}\left(1-\Delta_{\mathscr{S}}^{-2 l(\mathscr{S})}\right) \mathscr{Z}\left(\alpha, \alpha^{\prime}\right)=\left[1+\sum_{\mathscr{F}} \prod_{\mathscr{S} \in \mathscr{F}}\left(-\Delta_{\mathscr{S}}^{-2 l(\mathscr{\mathscr { S }})}\right)\right] \mathscr{Z}\left(\alpha, \alpha^{\prime}\right)
$$

where we sum over all forests $\mathscr{F}$ of subgraphs.

Proof. As we know from Ref. [13] (Appendix B), the proof is based upon the following equality

$$
\ldots\left(1-\Delta_{\cup \mathscr{S}_{2}}^{-2 l\left(\cup \mathscr{S}_{i}\right)}\right)\left(-\Delta_{\mathscr{S}_{1}}^{-2 l\left(\mathscr{S}_{1}\right)}\right) \ldots\left(-\Delta_{\mathscr{S}_{n}}^{-2 l\left(\mathscr{S}_{n}\right)}\right) \mathscr{Z}\left(\alpha, \alpha^{\prime}\right)=0,
$$

where the subgraphs $\mathscr{S}_{1}, \ldots, \mathscr{S}_{n}$ are disjoint. We dilate the variables $\alpha_{a}^{\prime}$ and $\left(\alpha_{a}, \alpha_{a}^{\prime}\right)$ for $a \in \mathscr{S}_{i}$ respectively by $\varrho_{i}^{\prime 2}$ and $\varrho_{i}^{2}$, and for $a \in \bigcup_{i=1}^{n} \mathscr{S}_{i}$ by $\varrho^{\prime 2}$ and $\varrho^{2}$. Then, $\mathscr{Z}\left(\alpha, \alpha^{\prime}\right)$ becomes $\mathscr{Z}\left(\varrho \varrho^{\prime} \varrho_{i} \varrho_{i}^{\prime}, \varrho \varrho_{i}, \alpha, \alpha^{\prime}\right)$. We expand the function $\mathscr{Z}$ in the variables $\varrho \varrho_{i}$ and $\varrho \varrho^{\prime} \varrho_{i} \varrho_{i}^{\prime}$ as

$$
\begin{aligned}
\mathscr{Z}\left(\varrho \varrho^{\prime} \varrho_{i} \varrho_{i}^{\prime}, \varrho \varrho_{i}, \alpha, \alpha^{\prime}\right)= & \prod_{i=1}^{n}\left(\varrho \varrho^{\prime} \varrho_{i} \varrho_{i}^{\prime}\right)^{-4 l\left(\mathscr{S}_{i}\right)-d\left(\mathscr{S}_{i}\right)} \\
& \cdot\left\{\sum_{k_{i}, k_{i}^{\prime}=0}^{\infty} \prod_{i=1}^{n}\left(\varrho \varrho_{i}\right)^{k_{i}} \prod_{i=1}^{n}\left(\varrho \varrho^{\prime} \varrho_{i} \varrho_{i}^{\prime}\right)^{k_{i}^{\prime}} \mathscr{Z}_{\left\{k, k^{\prime}\right\}}\left(\alpha, \alpha^{\prime}\right)\right\} .
\end{aligned}
$$

In (B.11), $L\left(\mathscr{S}_{i}\right)$ and $d\left(\mathscr{S}_{i}\right)$ are respectively the number of loops and of derivative couplings of the graph $\mathscr{S}_{i}$; we note that

$$
L\left(\bigcup_{i=1}^{n} \mathscr{S}_{i}\right)=\sum_{i=1}^{n} L\left(\mathscr{S}_{i}\right)
$$

and

$$
d\left(\bigcup_{i=1}^{n} \mathscr{S}_{i}\right)=\sum_{i=1}^{n} d\left(\mathscr{S}_{i}\right) .
$$


If we apply the operator $\Delta_{\mathscr{S}_{i}}^{-2 l\left(\mathscr{S}_{i}\right)}$ upon $\mathscr{Z}\left(\alpha, \alpha^{\prime}\right)$, two kind of terms are left in (B.11); the terms obtained after application of $\tau_{\mathscr{S}_{i}}^{-2 l\left(\mathscr{S}_{2}\right)-1}\left(\alpha^{\prime}\right)$ are such that $k_{i}^{\prime}<\omega\left(\mathscr{S}_{i}\right)-1$ where $\omega(\mathscr{S})$ is the superficial degree of divergence of $\mathscr{S}$; the terms obtained after application of $\tau_{\mathscr{S}_{i}}^{-2 l\left(\mathscr{S}_{i}\right)}\left(\alpha, \alpha^{\prime}\right)\left[1-\tau_{\mathscr{S}_{i}}^{-2 l\left(\mathscr{S}_{2}\right)-1}\left(\alpha^{\prime}\right)\right]$ are such that $k_{i}^{\prime}=\omega\left(\mathscr{S}_{i}\right)$ and $k_{i}=0$. To apply the operator $\left(1-\Delta_{\mathscr{S}}^{-2 l(\mathscr{S})}\right)$ is equivalent to the successive application of the operators $\left[1-\tau_{\mathscr{S}}^{-2 l(\mathscr{S})-1}\left(\alpha^{\prime}\right)\right]$ and $\left[1-\tau_{\mathscr{S}}^{-2 l(\mathscr{S})}\left(\alpha, \alpha^{\prime}\right)\right]$. The first operator applied with $\mathscr{S}=\bigcup_{i=1}^{n} \mathscr{S}_{i}$ tells that $\sum_{i=1}^{n} k_{i}^{\prime}>\omega\left(\bigcup_{i=1}^{n} \mathscr{S}_{i}\right)-1$, which is satisfied only if all $k_{i}^{\prime}=\omega\left(\mathscr{S}_{i}\right)$ and all $k_{i}=0$; the second operator requires $\sum_{i=1}^{n}\left(k_{i}+k_{i}^{\prime}\right)>\omega\left(\bigcup_{i=1}^{n} \mathscr{S}_{i}\right)$, which cannot be fulfilled. This proves Equation (B.10).

\section{The Forest Formula of One-Line Irreducible Connected Subgraphs}

\section{Theorem.}

$$
\prod_{\mathscr{S} \subseteq G}\left(1-\Delta_{\mathscr{S}}^{-2 l(\mathscr{S})}\right) \mathscr{Z}\left(\alpha, \alpha^{\prime}\right)=\left[1+\sum_{\mathscr{F}} \prod_{\mathscr{S} \in \mathscr{F}}\left(-\Delta_{\mathscr{S}}^{-2 l(\mathscr{S})}\right)\right] \mathscr{Z}\left(\alpha, \alpha^{\prime}\right),
$$

where we sum over all forests $\mathscr{F}$ of one line irreducible subgraphs.

Proof. In Ref. [13] (Appendix B), we show that the proof is based upon the following equality:

$$
\ldots\left(1-\Delta_{\mathscr{S}^{\prime}}^{-2 l\left(\mathscr{S}^{\prime}\right)}\right)\left(-\Delta_{\mathscr{S}}^{-2 l(\mathscr{S})}\right) \mathscr{Z}\left(\alpha, \alpha^{\prime}\right)=0,
$$

where $\mathscr{S}$ is a one-line reducible subgraph and $\mathscr{S}^{\prime}$ its one-line irreducible component. We dilate the variables $\alpha_{a}^{\prime}$ and $\left(\alpha_{a}, \alpha_{a}^{\prime}\right)$ for $a \in \mathscr{S}$ respectively by $\varrho^{\prime 2}$ and $\varrho^{2}$, and for $a \in \mathscr{S}^{\prime}$ by $\mu^{\prime 2}$ and $\mu^{2}$. Then $\mathscr{Z}\left(\alpha, \alpha^{\prime}\right)$ can be expanded as [14]

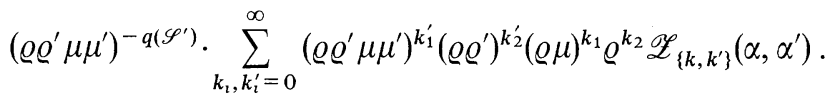

Then, the application of $\Delta_{\mathscr{S}}^{-2 l(\mathscr{S})}$ requires $k_{1}^{\prime}+k_{2}^{\prime} \leqq q\left(\mathscr{S}^{\prime}\right)-2 l(\mathscr{S})-1$, or $k_{1}^{\prime}+k_{2}^{\prime}=$ $q\left(\mathscr{S}^{\prime}\right)-2 l(\mathscr{S})$, with $k_{1}+k_{2}=0$; on the other hand, the application of

$$
\left[1-\tau_{\mathscr{S}^{\prime}}^{-2 l\left(\mathscr{P}^{\prime}\right)-1}\left(\alpha^{\prime}\right)\right]
$$

require $k_{1}^{\prime}>q\left(\mathscr{S}^{\prime}\right)-2 l\left(\mathscr{S}^{\prime}\right)>q(\mathscr{S})-2 l(\mathscr{S})$. That proves $(\mathrm{B} .15)$.

\section{Theorem.}

$$
\prod_{\mathscr{S} \cong G}\left(1-\Delta_{\mathscr{S}}^{-2 l(\mathscr{S})}\right) \mathscr{Z}\left(\alpha, \alpha^{\prime}\right)=\left[1+\sum_{\mathscr{F}} \prod_{\mathscr{S} \in \mathscr{F}}\left(-\Delta_{\mathscr{S}}^{-2 l(\mathscr{S})}\right)\right] \mathscr{Z}\left(\alpha, \alpha^{\prime}\right),
$$

where we sum over all forests of one-line irreducible, connected subgraphs.

Proof. In Ref. [13] (Appendix B), we show that the proof is based upon the following equality:

$$
\ldots\left(1-\Delta_{\mathscr{S}_{1}}^{-2 l\left(\mathscr{S}_{1}\right)}\right) \ldots\left(1-\Delta_{\mathscr{S}_{n}}^{-2 l\left(\mathscr{S}_{n}\right)}\right)\left(\begin{array}{c}
-2 l\left(\bigcup_{i=1}^{n} \mathscr{S}_{i}\right) \\
-\bigcup_{i=1}^{n} \mathscr{S}_{i}
\end{array}\right) \mathscr{Z}\left(\alpha, \alpha^{\prime}\right)=0
$$

where $\mathscr{S}_{1}, \mathscr{S}_{2}, \ldots, \mathscr{S}_{n}$ are disjoint subgraphs. To prove (B.18), we use (B.11) and apply the $\Delta$ operators successively. 
4. The Forest Formula of One-Line Irreducible, Connected, Divergent Subgraphs Theorem.

$$
\prod_{\mathscr{S} \cong G}\left(1-\Delta_{\mathscr{S}}^{-2 l(\mathscr{S})}\right) \mathscr{Z}\left(\alpha, \alpha^{\prime}\right)=\left[1+\sum_{\mathscr{F}} \prod_{\mathscr{S} \in \mathscr{F}}\left(-\Delta_{\mathscr{S}}^{-2 l(\mathscr{S})}\right)\right] \mathscr{Z}\left(\alpha, \alpha^{\prime}\right),
$$

where we sum over all forests of one-line irreducible, connected, divergent subgraphs.

Proof. The proof is trivial since $\Delta_{\mathscr{S}}^{-2 l(\mathscr{S})} \mathscr{Z}\left(\alpha, \alpha^{\prime}\right)$ is nul if $\mathscr{S}$ is a convergent subgraph.

\section{The Forest Formula of Generalized Vertices}

\section{Theorem.}

$$
\prod_{\mathscr{S} \subseteq G}\left(1-\Delta_{\mathscr{S}}^{-2 l(\mathscr{S})}\right) \mathscr{Z}\left(\alpha, \alpha^{\prime}\right)=\left[1+\sum_{\mathscr{F}} \prod_{\mathscr{S} \in \mathscr{F}}\left(-\Delta_{\mathscr{S}}^{-2 l(\mathscr{S})}\right)\right] \mathscr{Z}\left(\alpha, \alpha^{\prime}\right),
$$

where we sum over all forests of divergent generalized vertices.

Proof. In Ref. [13] (Appendix B) we show that the proof is based upon the following equality

$$
\ldots\left(1-\Delta_{\mathscr{S}_{2}}^{-2 l\left(\mathscr{S}_{2}\right)}\right)\left(-\Delta_{\mathscr{S}_{1}}^{-2 l\left(\mathscr{S}_{1}\right)}\right) \mathscr{Z}\left(\alpha, \alpha^{\prime}\right)=0,
$$

where $\mathscr{S}_{1}$ is a connected subgraph which is not a generalized vertex, and $\mathscr{S}_{2}$ is the generalized vertex obtained by adding $n$ lines to $\mathscr{S}_{1}$ but no vertices. Consequently, $\mathscr{S}_{2}$ has $n$ loops in addition to those of $\mathscr{S}_{1}$. We dilate the variables $\alpha_{a}^{\prime}$ and $\left(\alpha_{a}, \alpha_{a}^{\prime}\right)$ for $a \in \mathscr{S}_{1}$ by $\varrho^{\prime 2}$ and $\varrho^{2}$, and for $a \in \mathscr{S}_{2}$ by $\mu^{\prime 2}$ and $\mu^{2}$. Then, after dilatation of its variables, the function $\mathscr{Z}\left(\alpha, \alpha^{\prime}\right)$ can be expanded [14] as

$$
\begin{aligned}
& \left(\varrho \varrho^{\prime}\right)^{-4 L\left(\mathscr{S}_{1}\right)-d\left(\mathscr{S}_{1}\right)}\left(\mu \mu^{\prime}\right)^{-4 L\left(\mathscr{S}_{2}\right)-d\left(\mathscr{S}_{2}\right)} \\
& \cdot\left\{\sum_{k_{2}, k_{i}^{\prime}=0}^{\infty}\left(\varrho \varrho^{\prime} \mu \mu^{\prime}\right)^{k^{\prime}}\left(\varrho \varrho^{\prime}\right)^{k_{2}^{\prime}}(\varrho \mu)^{k_{1}} \mu^{k_{2}} \mathscr{Z}_{\left\{k, k^{\prime}\right\}}\left(\alpha, \alpha^{\prime}\right)\right\} .
\end{aligned}
$$

The application of $\Delta_{\mathscr{S}_{1}}^{-2 l\left(\mathscr{S}_{1}\right)}$ upon (B.22) implies $k_{1}^{\prime}+k_{2}^{\prime} \leqq \omega\left(\mathscr{S}_{1}\right)-1$, or $k_{1}^{\prime}+k_{2}^{\prime}=$ $\omega\left(\mathscr{S}_{1}\right)$ with $k_{1}=0$. The application of $\left(1-\tau_{2}^{-2 l\left(\mathscr{S}_{2}\right)-1}\left(\alpha^{\prime}\right)\right)$ in $\left(1-\Delta_{\mathscr{S}_{2}}^{-2 l\left(\mathscr{S}_{2}\right)}\right)$ implies $k_{1}^{\prime} \geqq \omega\left(\mathscr{S}_{2}\right)>\omega\left(\mathscr{I}_{1}\right)$. That proves (B.21). This theorem ensures that the new subtraction scheme satisfies the recurrence of Bogolubov and Parasiuk [7], and Hepp [8] (see Ref. [13], Section V). Similar results as those of paragraphs $3^{\circ}$ ), $4^{\circ}$ ), and $\left.5^{\circ}\right)$ can be also obtained from the complete product of $(1-\Delta)$ 's:

$$
\prod_{\mathscr{S} \cong G}\left(1-\Delta_{\mathscr{\mathscr { S }}}^{-2 l(\mathscr{S})}\right) \mathscr{Z}\left(\alpha, \alpha^{\prime}\right)=\prod_{\mathscr{S}^{\prime} \cong G}^{\prime}\left(1-\Delta_{\mathscr{S}^{\prime}}^{-2 l\left(\mathscr{S}^{\prime}\right)}\right) \mathscr{Z}\left(\alpha, \alpha^{\prime}\right),
$$

where the product on the right hand side runs only over divergent generalized vertices. Such a reduced form turns out to be useful for practical computation of the renormalized integrand.

\section{References}

1. Callan, C. G.: Phys. Rev. D2, 1541 (1970)

2. Symanzik, K.: Commun. math. Phys. 18, 227 (1970)

3. Lowenstein, J. H. : Commun. math. Phys. 24, 1 (1971) 
4. Gell-Mann, M., Low, F.: Phys. Rev. 95, 1300 (1954)

5. Bergère, M.C., Lam, Y.M.P.: Commun. math. Phys. 39, 1 (1974); and Asymptotic Expansion of Feynman amplitudes, Part II, The divergent case. Freie Universität Berlin, HEP May 74/9

6. Lowenstein, J. H., Zimmermann, W.: Commun. math. Phys. 44, 73 (1975)

7. Bogolubov, N. N., Parasiuk, O.: Acta Math. 97, 227 (1957)

Bogolubov, N. N., Shirkov, D. W.: Introduction to the theory of quantized fields. New York: Interscience Publ. 1959

8. Hepp, K.: Commun. math. Phys. 2, 301 (1966)

9. Symanzik, K.: Progr. Theoret. Phys. 20, 690 (1958)

10. Bergère, M. C., Zuber, J. B. : Commun. math. Phys. 35, 113 (1974)

11. Zimmermann, W.: Ann. Phys. 77, 536 (1973)

12. Weinberg, S.: Phys. Rev. 118, 838 (1960)

13. Bergère, M.C., Lam,Y.M.P.: Bogolubov Parasiuk theorem in the $\alpha$-parametric representation, Saclay preprint, DPh.T/75/89

14. Bergère, M.C., Lam,Y.M.P.: Lectures ou Renormalization Techniques in the $\alpha$-parametric representation, Course given in Saclay (January 1975), to be published

Communicated by K. Symanzik

Received October 30, 1975 